\title{
Chemical analysis of 24 dusty (pre-)main-sequence stars ${ }^{\star}$
}

\author{
B. Acke and C. Waelkens
}

\author{
Instituut voor Sterrenkunde, Katholieke Universiteit Leuven, Celestijnenlaan 200B, 3001 Leuven, Belgium \\ e-mail: bram.acke@ster. kuleuven. ac. be
}

Received 14 June 2004 / Accepted 6 August 2004

\begin{abstract}
We have analysed the chemical photospheric composition of 24 Herbig Ae/Be and Vega-type stars in search for the $\lambda$ Bootis phenomenon. We present the results of the elemental abundances of the sample stars. Some of the stars were never before studied spectroscopically at optical wavelengths. We have determined the projected rotational velocities of our sample stars. Furthermore, we discuss stars that depict a (selective) depletion pattern in detail. HD 4881 and HD 139614 seem to display an overall deficiency. AB Aur and possibly HD 126367 have subsolar values for the iron abundance, but are almost solar in silicon. HD 100546 is the only clear $\lambda$ Bootis star in our sample.
\end{abstract}

Key words. stars: abundances - stars: chemically peculiar - stars: planetary systems: protoplanetary disks stars: pre-main-sequence - stars: individual: HD 100546

\section{Introduction}

Herbig Ae/Be (HAEBE) stars are generally thought to be the somewhat more massive analogues of the T Tauri stars, which are low-mass pre-main-sequence stars. They were first defined as a class in 1960 by Herbig, based on three observational criteria: the stars have spectral type A or earlier, display emission lines, and illuminate a bright reflection nebula in their immediate vicinity. Later on other observational criteria were proposed. Finkenzeller \& Mundt (1984) mentioned that a shared characteristic of Herbig Ae/Be stars is the presence of an IR excess, which is an independent, but more easily detected signature of circumstellar matter. Following Thé et al. (1994) a useful working definition is: Herbig Ae/Be stars have spectral type $B$ to $F 8$ and luminosity class III to $V$, and show emission lines. They display a flux excess in the IR, due to the presence of cool and/or hot circumstellar matter. The observational properties of HAEBE stars have been reviewed by e.g. Pérez \& Grady (1997) and Waters \& Waelkens (1998).

That HAEBE stars are young objects has been convincingly confirmed by their presence in star-forming regions and also by the location in the Hipparcos HR diagram of individual objects (e.g., van den Ancker et al. 1998). Some of the objects often classified as HAEBE stars may be young main-sequence stars rather than pre-main-sequence stars, which is not really surprising, since, the more massive the star, the closer the circumstellar dissipation timescale approaches the pre-mainsequence lifetime. Dusty IR excesses, but without accompanying gaseous emission lines, are also observed in slightly or more evolved main-sequence stars, of which $\beta$ Pictoris and Vega are the most well known examples. The generic link

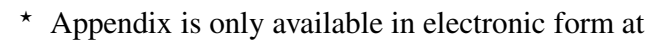
http://www. edpsciences.org between these debris-disk stars and HAEBE stars is a subject of current research to which we aim to contribute with the present paper.

Vega is the prototype of the Vega-like stars and shows mild characteristics of the so-called $\lambda$ Bootis stars, which are chemically peculiar A/F main sequence stars: the elements $\mathrm{C}, \mathrm{N}$, $\mathrm{O}$ and $\mathrm{S}$ have approximately solar photospheric abundances, while several metals are depleted. Venn \& Lambert (1990) pointed out that the $\lambda$ Boo abundance pattern is comparable to the composition of the gas component of the interstellar medium (e.g., Heiter 2002): the depleted elements (Mg, Ca, $\mathrm{Ti}, \mathrm{Fe}$ and $\mathrm{Sr}$ ) are those with the higher condensation temperatures. They suggested that the peculiar abundances could arise from gas/dust separation in the circumstellar environment, followed by accretion of the metal depleted gas. Reaccretion of circumstellar gas which is "cleaned" by this mechanism explains the most peculiar chemical composition of several postAGB stars, for which the depletion pattern is much more pronounced than in the $\lambda$ Bootis stars (e.g., Lambert et al. 1988; Waters et al. 1992). These peculiar post-AGB stars all turn out to be members of binaries, and the circumstellar dust occurs in a long-lived disk which surrounds the binary system (e.g., van Winckel et al. 1995; Waters et al. 1998; Molster et al. 1999).

Observational evidence (e.g., Mannings \& Sargent 1997; Testi et al. 2003; Piétu et al. 2003; Fuente et al. 2003; Natta et al. 2004) shows that the circumstellar matter in the vicinity of HAEBE stars displays a disk-like geometry, the modelling of which currently is the subject of intense research (e.g. disks powered by accretion (Lin \& Papaloizou 1980; Bell \& Lin 1994), irradiated passive disks (Kenyon \& Hartmann 1987), irradiated flared disks (Chiang \& Goldreich 1997), disks with an inner hole and a puffed up inner rim 
Table 1. The program stars, listed according to their HD numbers. The effective temperature and gravity of the Kurucz model used in the present abundance analysis. The last column indicates the category to which the objects belong. References: ${ }^{a}$ Backman \& Paresce (1993), ${ }^{b}$ Bhatt \& Manoj (2000), ${ }^{c}$ Coulson et al. (1998), ${ }^{d}$ Kalas et al. (2002), ${ }^{e}$ Malfait et al. (1998a), ${ }^{f}$ Patten \& Willson (1991), ${ }^{g}$ Song et al. (2000), ${ }^{h}$ Thé et al. (1994).

\begin{tabular}{|c|c|c|c|c|}
\hline \multicolumn{5}{|c|}{ Program stars } \\
\hline \multicolumn{2}{|c|}{ Object } & \multirow{2}{*}{$\begin{array}{c}T_{\mathrm{e}} \\
{[\mathrm{K}]}\end{array}$} & \multirow{2}{*}{$\begin{array}{c}\log g \\
{[\log \mathrm{cm} / \mathrm{s}]}\end{array}$} & \multirow[t]{2}{*}{ Cat. } \\
\hline HD & Other & & & \\
\hline HD 4881 & HR 241 & 11000 & 4.5 & $\mathrm{VEGA}^{d}$ \\
\hline HD 6028 & HR 287 & 8750 & 3.5 & VEGA $^{c}$ \\
\hline HD 17081 & $\pi$ Cet & 13000 & 4.0 & $\mathrm{HAEBE}^{e}$ \\
\hline HD 17206 & $\tau_{1}$ Eri & 6500 & 4.0 & VEGA $^{b}$ \\
\hline HD 18256 & $\rho$ Ari & 6750 & 4.5 & Check Star \\
\hline HD 20010 & $\alpha$ For & 6500 & 4.5 & VEGA $^{b}$ \\
\hline HD 28978 & HR 1448 & 9250 & 4.0 & $\mathrm{VEGA}^{a}$ \\
\hline HD 31293 & AB Aur & 9750 & 5.0 & $\mathrm{HAEBE}^{h}$ \\
\hline HD 31648 & & 8750 & 4.0 & HAEBE $^{h}$ \\
\hline HD 33564 & HR 1686 & 6250 & 4.0 & VEGA $^{f}$ \\
\hline HD 36112 & & 7750 & 3.5 & HAEBE $^{h}$ \\
\hline HD 95418 & $\beta \mathrm{UMa}$ & 9750 & 4.0 & VEGA $^{b, g}$ \\
\hline HD 97048 & CU Cha & 10000 & 4.0 & HAEBE $^{h}$ \\
\hline HD 97633 & $\theta$ Leo & 9250 & 3.5 & Check Star \\
\hline HD 100453 & & 7500 & 4.0 & $\mathrm{HAEBE}^{e}$ \\
\hline HD 100546 & KR Mus & 11000 & 4.5 & $\mathrm{HAEBE}^{h}$ \\
\hline HD 102647 & $\beta$ Leo & 8500 & 4.0 & VEGA $^{g}$ \\
\hline HD 104237 & DX Cha & 8000 & 4.5 & HAEBE $^{h}$ \\
\hline HD 135344 & & 6750 & 4.0 & $\mathrm{HAEBE}^{h}$ \\
\hline HD 139614 & & 8000 & 4.5 & $\mathrm{HAEBE}^{e}$ \\
\hline HD 190073 & & 9250 & 3.5 & $\mathrm{HAEBE}^{h}$ \\
\hline HD 244604 & & 8750 & 4.0 & $\mathrm{HAEBE}^{h}$ \\
\hline HD 245185 & & 9000 & 4.0 & HAEBE $^{h}$ \\
\hline HD 250550 & & 11000 & 4.0 & HAEBE $^{h}$ \\
\hline
\end{tabular}

(Dullemond et al. 2001; Dullemond 2002; Dullemond \& Dominik 2004). From the observation of redshifted resonance lines in the spectra of HAEBE stars, evidence for matter accretion has been found. These stars then seem natural candidates for displaying the $\lambda$ Bootis phenomenon. With the aim of further exploring the possible evolutionary connection between the Herbig Ae/Be stars and the Vega-type stars, we have carried out a chemical analysis of a selection of 24 objects, consisting of confirmed HAEBE stars and debris-disk stars and other objects which may be of interest in this context.

The plan of the paper is as follows: in the next section we describe the sample and the observations; Sect. 3 is devoted to the general results from the chemical analysis and a specific discussion of the objects for which depletion may occur. In Sect. 4 we discuss the results. In the Appendix, some notes on the individual objects are summarized, as well as a comparison with literature results.

\section{Observations and analysis}

We have obtained spectra for the 24 objects which are listed in Table 1. Fourteen program stars are HAEBE stars, eight objects are confirmed Vega-type stars or occur in lists of Vegatype candidates, and two stars (HD 18256 and HD 97633) are rather normal main-sequence A-type stars which we measured to check our analysis with respect to literature results. A first set of observations, concerning mostly the northern-hemisphere objects, was carried out by Drs. Hans van Winckel and Gwendolyn Meeus during the nights of 23 and 25 December 1996, with the William Herschel Telescope and the Utrecht Echelle Spectrograph at La Palma Observatory (Canary Islands, Spain). The second observational run, concerning mostly southern-hemisphere objects, was performed by Dr. Gwendolyn Meeus from January 26 till January 28, 1999 at the ESO site La Silla (Chile). These data were collected using the $1.5 \mathrm{~m}$ telescope and the FEROS instrument. The wavelength range of the spectra lies between 3860 and $9110 \AA$. The spectral resolution of both instruments is comparable $(\mathscr{R} \sim 45000)$. We reduced the data with the MIDAS software package.

Despite the broad wavelength coverage, for several program stars only a few lines turned out to be appropriate for detailed abundance analyses. Such a circumstance is not unusual for A/B-type stars, where the condition that spectral lines with equivalent widths higher than $150 \mathrm{~m} \AA$ should be avoided in abundance analyses and the fairly high projected rotational velocities severely limit the amount of suitable lines. For a few slowly rotating stars (e.g. HD 18256, HD 20010, HD 104327 and HD 139614) we were able to use hundreds of lines in the analysis; the average value for the amount of lines is about 100 . It has to be noted that the vast majority of usable lines are iron and titanium lines; our search for metal depletion primarily relies on these two elements, also on silicon and calcium for most objects, but on magnesium only in a minority of cases. On the other hand, for 6 program stars, no carbon, nitrogen and oxygen (CNO) abundances could be determined; the relative scarcity of CNO lines and the non-LTE (local thermodynamic equilibrium) sensitivity of especially nitrogen implies that the uncertainties on the CNO abundances are sometimes critical for assessing whether selective depletion occurs; in all cases at most a few lines are available for the determination of the sulphur abundance.

As starting values for the stellar parameters (effective temperature $T_{\mathrm{e}}$ and gravity $\log g$ ), we have used previously published results or values derived from photometric data. The equivalent widths derived from the spectra were converted to abundances using the program MOOG (Sneden 1973) for cooler stars $\left(T_{\mathrm{e}}<8500 \mathrm{~K}\right)$ and the Kurucz program WIDTH6 for hotter stars $\left(T_{\mathrm{e}}>8500 \mathrm{~K}\right)$, both programs supposing LTE. Non-LTE effects have not been taken into account. We have computed abundances with both programs for the stars with effective temperatures in the range from $7500 \mathrm{~K}$ and $9500 \mathrm{~K}$, and found good agreement. The stellar parameters were refined - when necessary and/or possible - in the usual way: the effective temperature was determined by asking that the abundances be independent from the excitation potential for ions with many observed lines; the gravity was set so that analyses for different ionisation stages for iron led to the same result. The stellar parameters as we adopted them in our abundance analyses are listed in Table 1. We were limited in chosing the effective temperature and gravity parameters by the finite grid of Kurucz models at our disposal - the grid has a step of $250 \mathrm{~K}$ in $T_{\mathrm{e}}$ 
Table 2. The solar abundances for C, N, O and S are respectively 8.56, 8.05, 8.93 and 7.21 dex (Anders \& Grevesse 1989). The given error represents the line-to-line scatter. The number of lines that were used in the abundance determination is mentioned in parentheses.

\begin{tabular}{|c|c|c|c|c|}
\hline \multicolumn{5}{|c|}{ CNO and $\mathrm{S}$ abundances } \\
\hline Object & {$[\mathrm{C}]$} & {$[\mathrm{N}]$} & {$[\mathrm{O}]$} & {$[\mathrm{S}]$} \\
\hline HD & dex & dex & dex & dex \\
\hline HD 4881 & $<-0.39(3)$ & $-0.49 \pm 0.05(4)$ & $-0.37 \pm 0.10(3)$ & \\
\hline HD 17081 & & & $-0.18 \pm 0.03(4)$ & $-0.07(1)$ \\
\hline HD 17206 & $-0.10 \pm 0.08(8)$ & $-0.16 \pm 0.02(2)$ & $-0.11 \pm 0.05(5)$ & $+0.00 \pm 0.14$ \\
\hline HD 18256 & $-0.10 \pm 0.08(7)$ & & & $-0.03 \pm 0.03(2)$ \\
\hline HD 20010 & $-0.32 \pm 0.16(11)$ & & & $-0.35 \pm 0.19(8)$ \\
\hline HD 28978 & $-0.20 \pm 0.01(2)$ & & $+0.00(1)$ & \\
\hline HD 31293 & & $+0.14(1)$ & $+0.21 \pm 0.07(2)$ & \\
\hline HD 31648 & $-0.23 \pm 0.05(4)$ & & & \\
\hline HD 33564 & $+0.30 \pm 0.12$ & & & $+0.10 \pm 0.01(2)$ \\
\hline HD 36112 & $-0.14 \pm 0.05$ & $-0.01(1)$ & & $+0.16(1)$ \\
\hline HD 95418 & & $-0.29 \pm 0.02(2)$ & $-0.33 \pm 0.04(3)$ & \\
\hline HD 97048 & & $+0.28(1)$ & $-0.11 \pm 0.14(6)$ & \\
\hline HD 97633 & & $-0.29 \pm 0.06(7)$ & $-0.33 \pm 0.01(2)$ & $+0.23 \pm 0.04(3)$ \\
\hline HD 100453 & $+0.00 \pm 0.09(7)$ & $-0.17(1)$ & & $-0.11 \pm 0.07(4)$ \\
\hline HD 100546 & & $+0.06(1)$ & $-0.05 \pm 0.10(10)$ & \\
\hline HD 104237 & $-0.14 \pm 0.20(30)$ & $-0.42 \pm 0.15(6)$ & $-0.10 \pm 0.14(11)$ & $-0.15 \pm 0.14(5)$ \\
\hline HD 135344 & $+0.16(1)$ & & & \\
\hline HD 139614 & $-0.06 \pm 0.14(21)$ & $-0.19 \pm 0.03$ & $-0.24 \pm 0.01(2)$ & $-0.22 \pm 0.04$ \\
\hline HD 190073 & $-0.07 \pm 0.03(3)$ & $+0.59(1)$ & $-0.27 \pm 0.07(4)$ & \\
\hline Vega & -0.10 & -0.05 & +0.08 & \\
\hline$\lambda$ Boo & -0.37 & +0.06 & -0.45 & \\
\hline ISM & -0.03 & -0.01 & -0.02 & \\
\hline
\end{tabular}

and a step of 0.5 dex in $\log g$. In each model, we have used a turbulent velocity of $v_{\text {tur }}=3 \mathrm{~km} \mathrm{~s}^{-1}$.

\section{Results of the chemical analysis}

\subsection{Overview}

In this section we present the derived elemental abundances. All values are given in reference to the (adopted) solar abundances given by Anders \& Grevesse (1989), $[X]=+0.0$ indicating a solar abundance for element $X$. Since we are investigating whether the selective depletion pattern is present in our sample stars, we compare the computed abundances with the elemental abundances of $\lambda$ Boo and Vega, two prototype $\lambda$ Bootis stars, and the interstellar medium (Paunzen et al. 1999; Venn \& Lambert 1990; Qiu et al. 2001; Erspamer $\&$ North 2002; Jenkins 1989). The stellar parameters for $\lambda$ Boo are $T_{\mathrm{e}}=8650 \mathrm{~K}$ and $\log g=4.00$ (Venn \& Lambert 1990) and for Vega $T_{\mathrm{e}}=9430 \mathrm{~K}$ and $\log g=3.95$ (Qiu et al. 2001). In Table 2 we have summarized the measured carbon, nitrogen, oxygen and sulphur abundances. Table 3 contains the abundances of the metals $\mathrm{Mg}, \mathrm{Si}, \mathrm{Ca}, \mathrm{Ti}, \mathrm{Fe}$ and $\mathrm{Sr}$. In Table 4, we have tabulated the abundances we computed for other elements. In parenthesis, the number of spectral lines that were used in the abundance determination are indicated ${ }^{1}$.

1 The complete linelist and measured equivalent widths can be downloaded at http://www.ster.kuleuven.ac.be/ bram/ LINELIST/linelist.ascii
All abundances tabulated in Tables $2-4$ and mentioned in the text have a line-to-line scatter smaller than $0.20 \mathrm{dex}$. The typical standard deviation is $\Delta \sim 0.10$ dex. The uncertainty on the stellar parameters introduces in general another $\sim 0.10 \mathrm{dex}$.

Lines - in several cases only one - of additional elements could be detected in the spectra of some program stars. The results are as follows. The He abundance of HD 250550 was determined to be $[\mathrm{He} \mathrm{I}]=-0.03$. For HD 20010 we computed $[\mathrm{Mn} \mathrm{II}]=+0.11 \mathrm{dex}$, which is in good agreement with the derived Mn I abundance. For HD 104237 we determined $[\mathrm{Al} \mathrm{II}]=+0.06 \mathrm{dex}$ and $[\mathrm{Cu} \mathrm{I}]=+0.26 \mathrm{dex}$ (for a solar abundance of $4.21 \mathrm{dex}$ ). For heavy elements, the following results were obtained: the LaII abundances for HD 17206, HD 100453 and HD 104237 are +2.07, +1.46 and +0.12 dex, respectively; the Ce abundances for HD 17206, HD 18256, HD 33564 and HD 139614 amount to $+1.26,+0.27,-0.15$ and +0.64 dex, respectively; for HD 17206, we found $[\mathrm{Nd} \mathrm{II}]=-0.05 \mathrm{dex}$, for HD $33564[\mathrm{Nd} \mathrm{II}]=+0.27 \mathrm{dex}$, and for HD 104237 [Nd II] $=+0.59 \mathrm{dex}$; the abundance of samarium in HD 104237 is +0.85 dex; the europium abundance of HD 17206 is +0.23 dex. The solar abundances of lanthanum, cerium, neodymium, samarium and europium we used, are respectively $1.22,1.55,1.50,1.00$ and $0.51 \mathrm{dex}$.

As a by-product of our spectral analysis, we have computed the projected rotational velocity of each star in our sample. The values are listed in Table 5. The typical error is of the order of $10 \%$. 
Table 3. The abundances of the "metals". The adopted solar abundances for Mg, Si, Ca, Ti, Fe and Sr are respectively 7.58, 7.55, 6.36, 4.99, 7.52 and 2.90 dex (Anders \& Grevesse 1989). The given error represents the line-to-line scatter. The number of lines that were used in the abundance determination is mentioned in parentheses.

\begin{tabular}{|c|c|c|c|c|c|c|c|c|c|c|c|}
\hline \multicolumn{12}{|c|}{ Metal abundances } \\
\hline $\begin{array}{c}\text { Object } \\
\text { HD }\end{array}$ & {$[\mathrm{Mg} \mathrm{I}]$} & {$[\mathrm{Mg}$ II] } & {$[\mathrm{SiI}]$} & [Si II] & [Ca I] & $\begin{array}{c}\text { [Ca II] } \\
\text { in dex }\end{array}$ & [Ti I] & [Ti II] & {$[\mathrm{Fe} I]$} & {$[\mathrm{Fe}$ II] } & {$[\mathrm{Sr}$ II] } \\
\hline HD 4881 & & $-0.70 \pm 0.05(3)$ & & $-1.15 \pm 0.07(5)$ & & & & $-0.49 \pm 0.14(12)$ & $-0.61 \pm 0.10(4)$ & $-0.72 \pm 0.18(19)$ & $-0.34 \pm 0.09(2)$ \\
\hline HD 6028 & & & & $-0.23 \pm 0.13(2)$ & & & & $+0.11 \pm 0.09$ (3) & $-0.13 \pm 0.01(3)$ & $-0.14 \pm 0.08(7)$ & \\
\hline HD 17081 & & $-0.11 \pm 0.02(4)$ & & $+0.05 \pm 0.28(4)$ & & & & $-0.18 \pm 0.13(7)$ & & $-0.26 \pm 0.19(29)$ & \\
\hline HD 17206 & $-0.15(1)$ & & $+0.23 \pm 0.10$ & $-0.04 \pm 0.04(3)$ & $+0.12 \pm 0.05$ & & & $-0.57(1)$ & $-0.06 \pm 0.18$ & $-0.10 \pm 0.13(13)$ & \\
\hline HD 18256 & $+0.07 \pm 0.01(2)$ & & $+0.09 \pm 0.11$ & $+0.03 \pm 0.02(2)$ & $+0.19 \pm 0.18(17)$ & & $+0.22 \pm 0.21$ & $+0.16 \pm 0.10(4)$ & $+0.05 \pm 0.13(115)$ & $+0.07 \pm 0.19(21)$ & \\
\hline HD 20010 & $-0.20 \pm 0.11(2)$ & & $-0.08 \pm 0.10$ & $-0.15 \pm 0.01(2)$ & $-0.10 \pm 0.17$ & & $-0.02 \pm 0.10(5)$ & $-0.06 \pm 0.02(3)$ & $-0.20 \pm 0.16(146)$ & $-0.12 \pm 0.17(27)$ & \\
\hline HD 28978 & $-0.35(1)$ & $-0.34(1)$ & & $+0.05 \pm 0.15(3)$ & $-0.06 \pm 0.05$ & & & $+0.21 \pm 0.18$ & $+0.17 \pm 0.14$ & $+0.20 \pm 0.16(29)$ & $+0.31 \pm 0.01(2)$ \\
\hline HD 31293 & & & & $-0.11 \pm 0.03(3)$ & & & & & $-1.04 \pm 0.12(2)$ & & \\
\hline HD 31648 & $-0.53(1)$ & & $+0.71(1)$ & & $+0.11(1)$ & & & & $+0.12(1)$ & $+0.01(1)$ & \\
\hline HD 33564 & & & & $+0.37 \pm 0.03(2)$ & $-0.13 \pm 0.10$ & & & $-0.10 \pm 0.23(28)$ & $-0.35 \pm 0.18(30)$ & $-0.41 \pm 0.19(13)$ & \\
\hline HD 36112 & & & $+0.07 \pm 0.11(3)$ & & $+0.05 \pm 0.09(8)$ & & & $-0.23 \pm 0.04(4)$ & $-0.12 \pm 0.13(32)$ & $-0.15 \pm 0.11$ & \\
\hline HD 95418 & $-0.40(1)$ & & & $+0.13 \pm 0.05(2)$ & & & & $+0.28(1)$ & $+0.21 \pm 0.07(7)$ & $+0.32 \pm 0.16(15)$ & \\
\hline HD 97048 & & & & $-0.75 \pm 0.06(2)$ & & & & & & & \\
\hline HD 97633 & & & & $+0.14 \pm 0.12(2)$ & $-0.09 \pm 0.12$ & & & $+0.01(1)$ & $+0.00 \pm 0.10$ & $+0.04 \pm 0.15$ & \\
\hline HD 100453 & & $-0.01 \pm 0.09(2)$ & $+0.13 \pm 0.07(5)$ & $+0.15 \pm 0.04(2)$ & $+0.03 \pm 0.12(16)$ & & & $-0.14 \pm 0.18(11)$ & $-0.10 \pm 0.19(81)$ & $-0.07 \pm 0.19(26)$ & \\
\hline HD 100546 & $-1.14 \pm 0.07(4)$ & & & $-0.84 \pm 0.13(6)$ & & & & & & $-1.30 \pm 0.13(5)$ & \\
\hline HD 102647 & & & & & $+0.12 \pm 0.14$ & & & & $-0.27 \pm 0.15(6)$ & $-0.18 \pm 0.11(4)$ & \\
\hline HD 104237 & $-0.12(1)$ & $-0.19 \pm 0.07(2)$ & $+0.02 \pm 0.11(14)$ & $+0.31 \pm 0.09(5)$ & $+0.15 \pm 0.12(21)$ & $+0.14(1)$ & & $+0.23 \pm 0.19$ & $+0.08 \pm 0.18(175)$ & $+0.10 \pm 0.17(56)$ & \\
\hline HD 135344 & & & $+0.21(1)$ & $-0.05 \pm 0.10(2)$ & $+0.00 \pm 0.27$ (4) & & & $-0.45 \pm 0.01(2)$ & $-0.07 \pm 0.10$ & $-0.09(1)$ & \\
\hline HD 139614 & $-0.48 \pm 0.06(2)$ & $-0.35 \pm 0.10(3)$ & $-0.36 \pm 0.04(2)$ & $-0.21 \pm 0.08(5)$ & $-0.24 \pm 0.10(17)$ & & & $-0.13 \pm 0.16(38)$ & $-0.30 \pm 0.15(116)$ & $-0.24 \pm 0.16$ & \\
\hline HD 190073 & $-0.06(1)$ & $-0.06 \pm 0.02(3)$ & & $+0.08 \pm 0.12(3)$ & $+0.29 \pm 0.09$ & & & $+0.04 \pm 0.13(26)$ & $+0.07 \pm 0.20$ & $-0.07 \pm 0.11(16)$ & \\
\hline HD 244604 & & & & & & & & & $-0.25(1)$ & & \\
\hline HD 250550 & & $-0.42(1)$ & & $-0.17 \pm 0.09(3)$ & & & & $+0.02(1)$ & & $-0.88 \pm 0.12(4)$ & \\
\hline Vega & -0.77 & -0.89 & & -0.59 & -0.95 & -1.34 & & -0.41 & -0.58 & -0.59 & -1.62 \\
\hline$\lambda$ Boo & -2.00 & -2.00 & & -1.00 & & -1.97 & & -2.03 & -2.05 & -2.05 & -2.10 \\
\hline ISM & -0.70 & -0.70 & & & -3.60 & -3.60 & & -2.70 & -2.10 & -2.10 & \\
\hline
\end{tabular}


Table 4. The abundances of other elements. The adopted solar abundances for $\mathrm{Na}, \mathrm{Al}, \mathrm{Sc}, \mathrm{V}, \mathrm{Cr}, \mathrm{Mn}, \mathrm{Ni}, \mathrm{Zn}, \mathrm{Y}, \mathrm{Zr}$ and $\mathrm{Ba}$ are respectively 6.33, 6.47, 3.10, 4.00, 5.67, 5.39, 6.25, 4.60, 2.24, 2.60 and 2.13 dex (Anders \& Grevesse 1989). The given error represents the line-to-line scatter. The number of lines that were used in the abundance determination is mentioned in parentheses. Two numbers refer to the neutral and first ionization state of the element, respectively.

\begin{tabular}{|c|c|c|c|c|c|c|c|c|c|c|c|}
\hline \multicolumn{12}{|c|}{ Other elemental abundances } \\
\hline $\begin{array}{c}\text { Object } \\
\text { HD }\end{array}$ & {$[\mathrm{NaI}]$} & [AlI] & [Sc II] & {$[\mathrm{V}]$} & {$[\mathrm{Cr}]$} & $\begin{array}{l}{[\mathrm{MnI}]} \\
\text { in } \operatorname{dex}\end{array}$ & {$[\mathrm{Ni}]$} & {$[\mathrm{ZnI}]$} & [Y II] & {$[\mathrm{ZrII}]$} & [Ba II] \\
\hline HD 4881 & & & & & $-0.64 \pm 0.10(0 / 9)$ & & & & & & \\
\hline HD 6028 & & $-0.48(1)$ & & & $+0.02 \pm 0.19(0 / 2)$ & & $+0.22(0 / 1)$ & & & & \\
\hline HD 17081 & & & & & $-0.21 \pm 0.08(0 / 10)$ & & $-0.10 \pm 0.02(0 / 2)$ & & & & \\
\hline HD 17206 & $+0.03 \pm 0.03(2)$ & $+0.05(1)$ & $-0.57(1)$ & & $-0.01 \pm 0.16(9 / 9)$ & $-0.04 \pm 0.08(3)$ & $+0.09 \pm 0.16(17 / 0)$ & $-0.35 \pm 0.15(2)$ & $+0.34 \pm 0.07(2)$ & $+0.77 \pm 0.02(2)$ & $-0.32 \pm 0.08(3)$ \\
\hline HD 18256 & $+0.13 \pm 0.01(2)$ & $-0.12 \pm 0.05(2)$ & $+0.17(1)$ & & $+0.22 \pm 0.12(9 / 10)$ & $+0.05(1)$ & $+0.09 \pm 0.19(23 / 0)$ & & $-0.04 \pm 0.09(3)$ & & $-0.47 \pm 0.05(2)$ \\
\hline HD 20010 & $-0.16 \pm 0.04(4)$ & $-0.47 \pm 0.13(4)$ & $+0.07 \pm 0.09(6)$ & & $-0.01 \pm 0.10(8 / 11)$ & $+0.12 \pm 0.21(4)$ & $-0.05 \pm 0.18(26 / 0)$ & $-0.14(1)$ & $-0.02(1)$ & & $+0.11 \pm 0.03(2)$ \\
\hline HD 28978 & & $-0.52(1)$ & $+0.18 \pm 0.15(5)$ & $+0.24 \pm 0.08(0 / 3)$ & $+0.06 \pm 0.08(2 / 17)$ & & $+0.30 \pm 0.14(2 / 3)$ & & $+0.49 \pm 0.07(3)$ & $+0.75 \pm 0.00(3)$ & $+0.67 \pm 0.03(3)$ \\
\hline HD 31648 & & & & & & & & & $+0.75(1)$ & & \\
\hline HD 33564 & $+0.60(1)$ & & $-0.21 \pm 0.10(5)$ & $-0.61(1 / 0)$ & $-0.21 \pm 0.12(5 / 9)$ & $-0.29 \pm 0.06(6)$ & $-0.13 \pm 0.11(11 / 2)$ & $-0.56 \pm 0.03(2)$ & $+0.05 \pm 0.11(3)$ & $+0.16 \pm 0.07(5)$ & $+0.69(1)$ \\
\hline HD 36112 & & & $-0.13(1)$ & & $-0.11 \pm 0.10(1 / 6)$ & $-0.16 \pm 0.15(2)$ & $-0.18(1 / 0)$ & & $+0.55 \pm 0.11(2)$ & & \\
\hline HD 95418 & & & & & $+0.49 \pm 0.06(0 / 4)$ & & & & & & \\
\hline HD 97633 & $+0.20 \pm 0.04(2)$ & & & & $+0.17 \pm 0.14(0 / 7)$ & & & & & & \\
\hline HD 100453 & $+0.14 \pm 0.14(3)$ & & $-0.21 \pm 0.03(3)$ & $+0.28(0 / 1)$ & $+0.03 \pm 0.10(3 / 6)$ & $-0.04 \pm 0.17(2)$ & $-0.03 \pm 0.15(7 / 0)$ & $-0.52(1)$ & & $+0.48 \pm 0.05(2)$ & $-0.02(1)$ \\
\hline HD 100546 & & & & & $-0.87 \pm 0.05(0 / 2)$ & & & & & & \\
\hline HD 104237 & $+0.07 \pm 0.10(4)$ & $-0.02 \pm 0.12(4)$ & $+0.08 \pm 0.05(9)$ & $-0.01 \pm 0.14(1 / 2)$ & $+0.17 \pm 0.13(16 / 24)$ & $-0.05 \pm 0.09(7)$ & $+0.09 \pm 0.14(34 / 0)$ & $-0.37 \pm 0.10(2)$ & $+0.18 \pm 0.10(8)$ & $+0.35 \pm 0.11(6)$ & $+0.31 \pm 0.05(2)$ \\
\hline HD 135344 & & & $-0.36(1)$ & & $+0.06 \pm 0.06(2 / 2)$ & & $+0.08 \pm 0.19(2 / 0)$ & $-0.45(1)$ & & & \\
\hline HD 139614 & & & $-0.21 \pm 0.08(5)$ & $-0.01 \pm 0.08(0 / 2)$ & $-0.20 \pm 0.08(11 / 19)$ & $-0.30 \pm 0.01(2)$ & $-0.18 \pm 0.16(13 / 0)$ & $-0.52 \pm 0.14(2)$ & $+0.04 \pm 0.18(3)$ & $+0.47 \pm 0.04(4)$ & $+0.06 \pm 0.01(2)$ \\
\hline HD 190073 & & $-0.45(1)$ & $-0.05 \pm 0.04(3)$ & $+0.09 \pm 0.08(3 / 0)$ & $+0.01 \pm 0.12(2 / 17)$ & & $+0.06 \pm 0.07(0 / 2)$ & & $+0.03(1)$ & $+0.31(1)$ & $+0.11(1)$ \\
\hline HD 250550 & & & & & $-0.05(0 / 1)$ & & $+0.06(0 / 1)$ & & & & \\
\hline Vega & +0.12 & & -0.77 & -0.26 & -0.48 & -0.30 & +0.02 & & -0.59 & -0.93 & -1.22 \\
\hline$\lambda$ Boo & -1.30 & & -0.70 & & -1.00 & & -1.20 & & & & -0.90 \\
\hline ISM & -0.50 & & & & & & & & & & \\
\hline
\end{tabular}




\subsection{Stars with evidence for (selective) depletion}

\subsubsection{HD 4881}

Lagrange-Henri et al. (1990) considered this star, which has a Vega-like IR excess, in a list of possible analogues to $\beta$ Pictoris in a search for redshifted $\mathrm{Ca}$ II and $\mathrm{Na}$ I absorption, which would point to accretion of circumstellar matter; it is not clear from their paper whether they observed the star. Miroshnichenko et al. (1999) investigated the optical and IR photometry, the $\mathrm{H} \alpha$ emission and measured equivalent widths of prominent spectral lines; they found that the star has a far IR excess, but rejected it as a Herbig Ae/Be star and classified it as a classical Be star. Kalas et al. (2002) discovered a Pleiades-like nebulosity around HD 4881, but concluded that there was possibly still need for a second IR source to explain the IRAS fluxes; they suggested that this extra source consists of warm grains, either in a circumstellar disk or in a broader region where nonequilibrium small-grain heating occurs.

We observed HD 4881 in the spectral region from 3860 to $5020 \AA$ and from 5220 to $9100 \AA$. A few strong spectral lines Mg II $\lambda 4481$, Si II $\lambda \lambda 4128,4130,6347,6371$ and Ca II $\lambda 3933-$ in the our spectrum have a peculiar shape (see Fig. 1). The profile seems to be made up of two components - a superposition of a broad line and a narrower Gaussian line. The straightforward interpretation is that the narrow line is of circumstellar origin, while the broad line is the pressure broadened photospheric line. A strange coincidence is then that the broadness of the photospheric lines of the other elements in this deficient star is close to that of the narrow components of the strong lines. The projected rotational velocity we found for HD 4881 is $25 \mathrm{~km} \mathrm{~s}^{-1}$. Abt \& Morrell (1995) suggested a $v \sin i$ of $65 \mathrm{~km} \mathrm{~s}^{-1}$, in rather strong disagreement with our value. However, Abt $\&$ Morrell determined $v \sin i$ by fitting a Gaussian curve to the $\lambda 4481 \mathrm{Mg}$ II line, of which the profile is more complex than Gaussian.

Our effective temperature for the Kurucz model of HD $4881,11000 \mathrm{~K}$, is consistent with the $B-V$ color index of -0.07 (Kalas et al. 2002; Flower 1996) and spectral classification B9.5V. Higher effective temperatures lead to a discrepancy between the Fe I and the Fe II abundances. We thus disagree with Miroshnichenko et al. (1999) who proposed a spectral type B8 for HD 4881 and with the effective temperature of $12300 \mathrm{~K}$ derived by Kalas et al. (2002) from fitting the spectral energy distribution. Miroshnichenko et al. also determined the effective temperature on the basis of the Balmer jump and the $\mathrm{H} \alpha$ line, respectively $T_{\mathrm{e}}=10700 \pm 500 \mathrm{~K}$ and $11000 \mathrm{~K}$; in agreement with our determination.

The result of our abundance analysis of HD 4881 is that it is deficient in all elements we studied, i.e. this star reveals a complete depletion pattern. The iron abundance, measured from 4 Fe I lines and 19 Fe II lines, is about -0.70 dex. Similar deficiencies are found for titanium and chromium, from 12 and 9 lines respectively, and for the $\alpha$ element magnesium from 3 lines, while silicon ( 5 lines) appears still more deficient. With respect to the $\lambda$ Bootis phenomenon, however, we note that also for carbon, nitrogen and oxygen subsolar abundances were found.

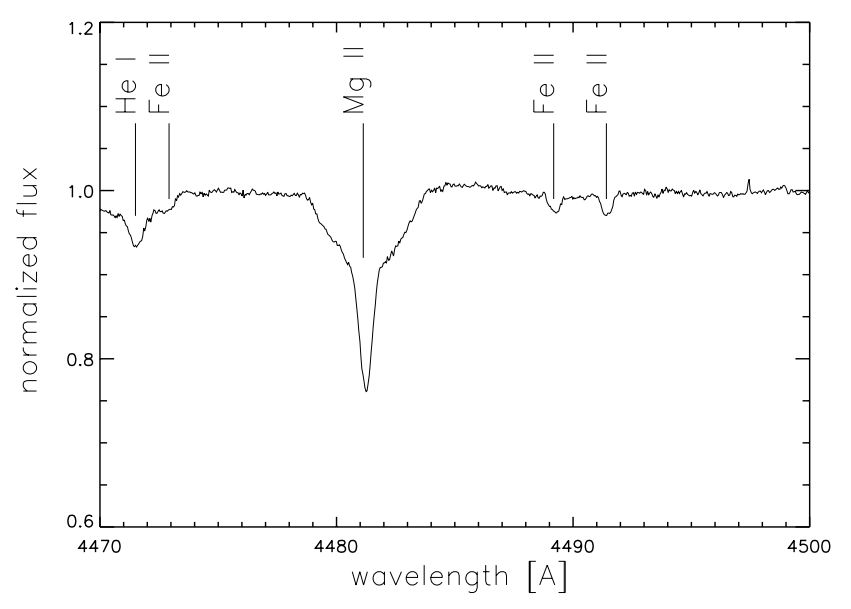

Fig. 1. The peculiar shape of the Mg II line near $4481 \AA$ in the spectrum of HD 4881. Four Si II lines (4128, 4130, 6347, $6371 \AA$ A) and a Ca II line (3933 ̊) show a similar profile.

Table 5. The projected rotational velocity.

\begin{tabular}{cc|cc}
\hline \hline \multicolumn{4}{c}{ Projected rotational velocity } \\
\hline $\begin{array}{c}\text { Star } \\
\text { HD }\end{array}$ & $\begin{array}{c}v \sin i \\
{\left[\mathrm{~km} \mathrm{~s}^{-1}\right]}\end{array}$ & $\begin{array}{c}\text { Star } \\
\text { HD }\end{array}$ & $\begin{array}{c}v \sin i \\
{\left[\mathrm{~km} \mathrm{~s}^{-1}\right]}\end{array}$ \\
\hline HD 4881 & 25 & HD 97048 & 140 \\
HD 6028 & 95 & HD 97633 & 20 \\
HD 17081 & 18 & HD 100453 & 39 \\
HD 17206 & 22 & HD 100546 & 55 \\
HD 18256 & 17 & HD 102647 & 120 \\
HD 20010 & 10 & HD 104237 & 10 \\
HD 28978 & 23 & HD 135344 & 69 \\
HD 31293 & 100 & HD 139614 & 24 \\
HD 31648 & 90 & HD 190073 & 9 \\
HD 33564 & 12 & HD 244604 & 90 \\
HD 36112 & 60 & HD 250550 & 54 \\
HD 95418 & 40 & & \\
\hline \multicolumn{4}{c}{}
\end{tabular}

An alternative explanation for the peculiar line shape observed in a number of - intrinsically strong - lines may be binarity. When both components have comparable effective temperatures, the spectrum of HD 4881 is the superposition of two spectra. Under this hypothesis, the line profile displayed in Fig. 1 contains a broad feature belonging to the component of the binary with the largest projected rotational velocity $\left(v \sin i \approx 100 \mathrm{~km} \mathrm{~s}^{-1}\right)$. The narrow absorption feature is then due to the second component, which also causes the other narrow photospheric absorption lines in the composite spectrum. The binary hypothesis potentially provides a solution for the current inconsistencies in determinations of the effective temperature and the projected rotational velocity of HD 4881. Furthermore, the observed overall chemical depletion pattern may be due to veiling effects. Although this hypothesis appears very attractive, no independent observational evidence for the binary nature of HD 4881 is available in the literature.

\subsubsection{AB Aur}

AB Aur (HD 31293) has been previously mentioned as a $\lambda$ Bootis candidate by Renson et al. (1990). Indeed, while 
we find slightly suprasolar values for the nitrogen and oxygen abundances, iron appears to be strongly depleted. Admittedly, the iron abundance is based on only 2 lines, but these lines provided reliable abundances for the other sample stars, and even taking into account the projected rotational velocity of $100 \mathrm{~km} \mathrm{~s}^{-1}$, it seems impossible to assign a solar iron abundance to this frequently studied HAEBE star. On the other hand, $\mathrm{AB}$ Aur appears to be only mildly deficient in silicon $([\mathrm{Si}]=-0.11 \mathrm{dex})$. The possible $\lambda$ Bootis nature of this object thus needs further investigation.

\subsubsection{HD 100546}

HD 100546 is one of the closest and brightest HAEBE stars. In IUE observations, Grady et al. (1995) detected redshifted absorption features from iron resonance lines reminiscent of those observed for $\beta$ Pictoris and concluded that also HD 100546 undergoes a bombardment by bodies of cometary size. The ISO (Infrared Space Observatory Kessler et al. 1996) spectrum of HD 100546 is spectacular, in the sense that it presents prominent crystalline silicate features with a spectrum closely resembling that of comet Hale-Bopp (Malfait et al. 1998b). The stellar parameters that were used in our Kurucz model for HD 100546 are taken from Meeus et al. (2001). The projected rotational velocity of HD 100546 is $55 \mathrm{~km} \mathrm{~s}^{-1}$, so that most lines in the optical spectrum should be suitable for abundance analysis purposes. However, it turns out that only a limited number of - intrinsically strong - lines could be measured accurately, and that the results consistently point to this star being deficient in several important elements. In fact, HD 100546 turns out to be the only clear $\lambda$ Bootis star in our sample, being deficient about 1 order of magnitude in magnesium (4 lines), silicon (6 lines) and iron (5 lines), but apparently having solar CNO abundances. Late B stars showing relatively few suitable CNO lines, the latter statement is essentially based on our determination of the oxygen abundance, which was estimated based on the measured equivalent widths of 10 spectral lines - amongst others six lines from the multiplets around $\lambda 6156$ and $\lambda 6454$ - which led to a consistent result with a scatter of 0.10 dex. The nitrogen abundance was computed using only the $7468 \AA$ N I line. The six Si II lines included the easily recognizable $6347 \AA$ and $6371 \AA$ lines; the five iron lines are those at $4233,4555,4583,5169$ and $5275 \AA$, the atomic parameters of which are quite reliable. A similar deficiency was found for chromium, where the lines at $\lambda 4558$ and $\lambda 4588$ were used to compute $[\mathrm{Cr}$ II $]=-0.87 \mathrm{dex}$.

\subsubsection{HD 139614}

The star HD 139614 is one of the objects in the sample for which the largest amount of lines can be studied, thanks to a small projected rotational velocity, which we found to be $24 \mathrm{~km} \mathrm{~s}^{-1}$, in agreement with Dunkin et al. (1997). According to Meeus et al. (1998), it is likely that the low $v \sin i$ is at least partly due to a near-pole-on inclination of the star. The stellar parameters for HD 139614 are again extracted from Meeus et al. (2001).

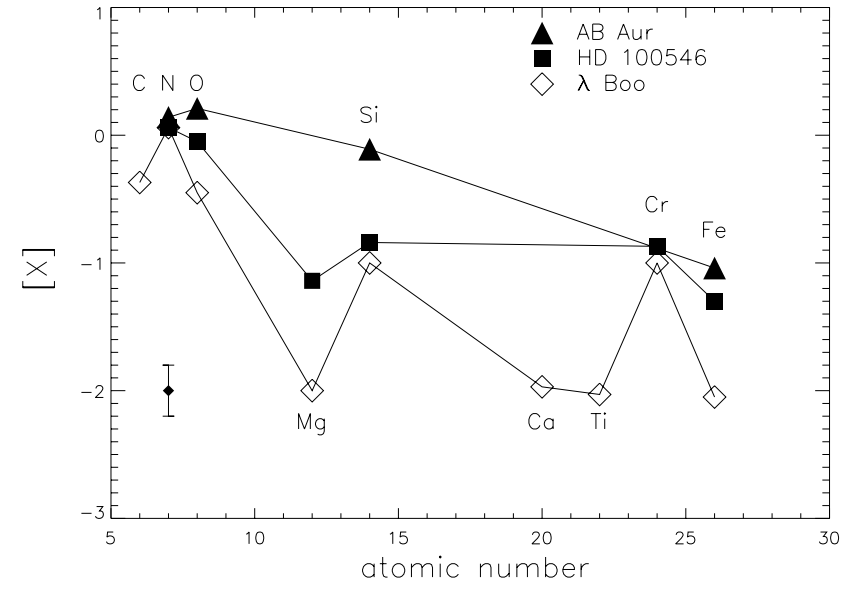

Fig. 2. The elemental abundances of AB Aur and HD 100546 determined in the present analysis compared to those of $\lambda$ Boo (Venn \& Lambert 1990). The depletion pattern of HD 100546 and $\lambda$ Boo are clearly similar. This is less obvious for AB Aur. In the lower left corner, the typical \pm 0.20 dex error bar is shown.

We found that all elements are slightly deficient ( -0.20 dex). The abundance pattern of this star is comparable to the pattern in HD 4881, but the deficiency is less strong. Dunkin et al. (1997) also examined HD 139614 spectroscopically. They used a higher effective temperature $T_{\mathrm{e}}=8250 \mathrm{~K}$ and a lower gravity $\log g=4.2$ in their model. They concluded that $[\mathrm{C}]=-0.07 \mathrm{dex},[\mathrm{Mg}]=-0.08 \mathrm{dex},[\mathrm{Si}]=-0.52 \mathrm{dex}$, $[\mathrm{S}]=-0.12 \mathrm{dex},[\mathrm{Ca}]=-0.33 \mathrm{dex},[\mathrm{Ti}]=-0.21 \mathrm{dex}$, $[\mathrm{Cr}]=-0.11 \mathrm{dex},[\mathrm{Fe}]=-0.03 \mathrm{dex}$ and $[\mathrm{Ni}]=-0.07 \mathrm{dex}$. The abundances of carbon, sulphur, calcium, titanium, chromium and nickel are in relatively good agreement with our values. The computed abundances of magnesium, silicon and iron differ from our results by at least 0.15 dex. We note that Dunkin et al. base their investigation mostly on strong lines, while we systematically discarded all lines with an equivalent width exceeding $150 \mathrm{~m} \AA$. In fact, of the respectively $116 \mathrm{Fe}$ I lines and $33 \mathrm{Fe}$ II lines we used in our analysis, only $1+4$ occur in the list of lines studied by Dunkin et al. (1997). It is not unlikely that our analysis is less affected by the uncertainties induced by microturbulence, so that we are confident that the small overall underabundance we found for HD 139614 is genuine.

In Fig. 2 the depletion pattern of AB Aur and HD 100546 is compared to the elemental-abundance pattern of $\lambda$ Boo. Figure 3 displays the overall-underabundance pattern of HD 4481 and HD 139614. No similarities with the elemental abundances of $\lambda$ Boo are noted in these sources.

\section{Discussion}

A few possible mechanisms have been proposed to explain the $\lambda$ Bootis abundance pattern. The selective-accretion theory of Venn \& Lambert (1990) is currently the most widely supported model. In the recent literature, Faraggiana \& Bonifacio (1999); Gerbaldi et al. (2003) and Faraggiana et al. (2004) mention that binarity can play an underestimated role. The composite spectrum might mimic the spectrum of a truly underabundant single star. Veiling due to the companion will make the metallic lines 


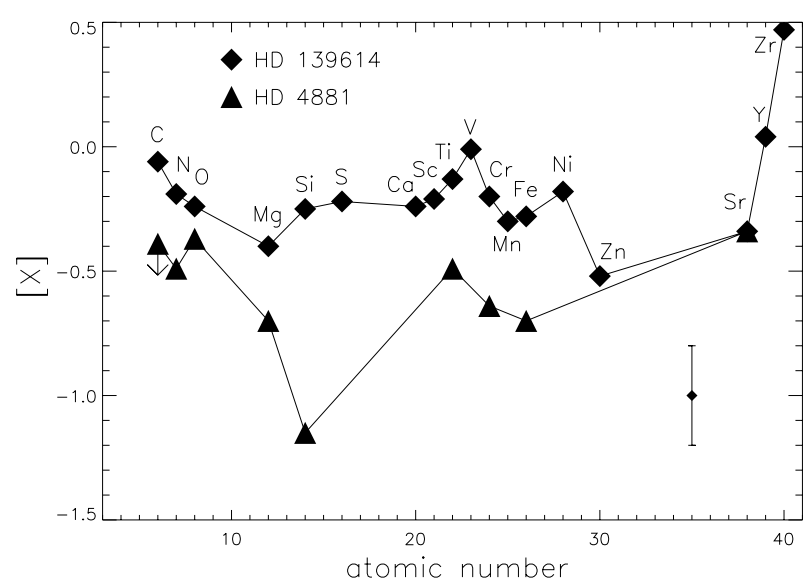

Fig. 3. The elemental abundances of HD 4881 and HD 139614 determined in the present analysis. Both patterns display an overall underabundance of -0.65 dex for HD 4881 and -0.22 dex for HD 139614 . In the lower right corner, the typical \pm 0.20 dex error bar is shown.

in the spectrum appear weaker. It is indeed not unlikely that some objects are misclassified as $\lambda$ Bootis stars, since the definition of this group of objects often used in the literature is " $\mathrm{A} / \mathrm{F}$ stars with weak metallic lines". We note however that the $\lambda$ Boo abundance pattern also requires solar $\mathrm{CNO}$ and $\mathrm{S}$ abundances. Veiling due to binarity works as much for the $\mathrm{CNO}$ and $\mathrm{S}$ absorption lines in the spectrum, hence making the measured equivalent widths of these lines lower than expected as well. The overall abundance pattern however remains roughly unaltered. It is therefore important that metallic as well as CNO and $\mathrm{S}$ abundances are determined to classify an object as a $\lambda$ Bootis star. We stress that the binarity of the selectively depleted postAGB stars cannot account for the observed abundances either. This can be deduced from the solar abundance $\mathrm{CNO}$ and $\mathrm{S}$, and zinc in these objects (e.g., van Winckel 2003).

Even though young stars with circumstellar (debris) disks are a priori fit environments for the selective accretion to take place (following Venn \& Lambert 1990), the typical abundance pattern is not present in the vast majority of these objects. Only one star in the present sample, HD 100546, appears to be selectively depleted. Because of the similarities of the abundance pattern of HD 100546 and $\lambda$ Boo, we suggest that accretion of metal-depleted gas is a plausible explanation for this source. Nevertheless, no other HAEBE or Vega-type star in our sample displays this behaviour. A few possible reasons can be proposed. Selective accretion might be a non-trivial process, which only occurs in a minority of the sources. One of the most stringent assumptions for the phenomenon to happen is the decoupling of the gas and dust particles. When closely interacting, the infalling, metal-depleted gas will drag the (metalrich) dust along. Both gas and dust will be accreted in this case, leaving the photospheric abundances of the central star unaltered. A second explanation for the lack of $\lambda$ Boo stars in this sample may be the timescales involved. The dust particles are more easily prevented from falling in, due to the larger radiation pressure. Nevertheless, also the dust is expected to eventually spiral in and be accreted. The depletion pattern is only present after the gas has been accreted, and before the dust falls in onto the stellar photosphere. The perceptibility

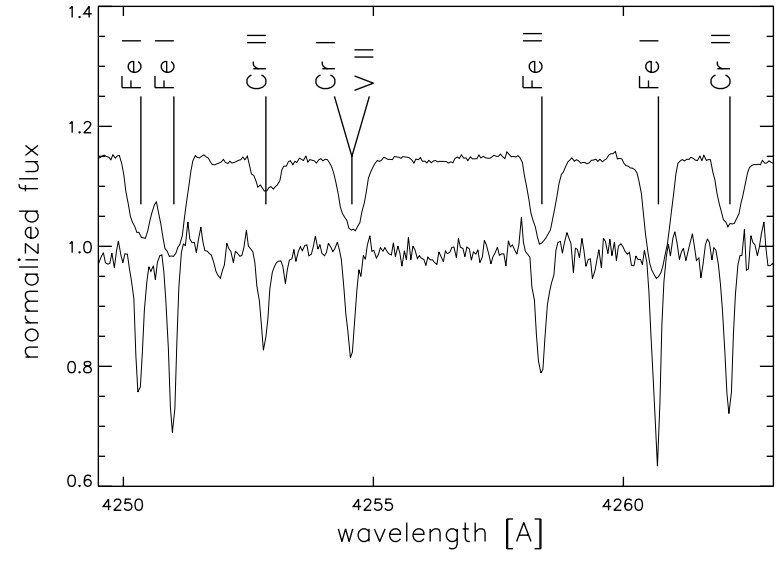

Fig. 4. The spectra of HD 28978 (top), an A2 star with $T_{\mathrm{e}}=9250 \mathrm{~K}$, and HD 190073 (bottom), are very similar. The agreement between both spectra confirms our lower - with respect to other authors - effective temperature for HD 190073.

of the $\lambda$ Bootis phenomenon would then depend on the difference in accretion timescale between gas and dust particles. Moreover, Turcotte \& Charbonneau (1993) have modeled the effect of stellar rotation and chemical diffusion on the photospheric abundance pattern. They find that a constant infall of at least $10^{-14} M_{\odot} \mathrm{yr}^{-1}$ is needed to maintain the depletion of the metals when photospheric diffusion is taken into account. After accretion of gas has stopped, the abundance pattern is destroyed on a timescale of $10^{6} \mathrm{yr}$. Stellar rotation and the consequent meridional circulation only plays a role for rotational velocities above $\sim 125 \mathrm{~km} \mathrm{~s}^{-1}$.

Imaging of HD 100546 shows that the inclination of the circumstellar disk around this source is $i \sim 50^{\circ}$ (Pantin et al. 2000; Augereau et al. 2001; Grady et al. 2001) where $i=0^{\circ}$ represents pole-on. Assuming that the rotational vector of the star and the disk are located along the same axis, the rotational velocity of HD 100546 is $v \approx 70 \mathrm{~km} \mathrm{~s}^{-1}$. This is consistent with Turcotte $\&$ Charbonneau (1993), since the $\lambda$ Bootis abundance pattern is observable. AB Aur on the other hand is a rapidly rotating star. Using the inclination of $i<30^{\circ}$ (Eisner et al. 2003; Blake \& Boogert 2004) of the circumstellar disk for the stellar rotation, the rotational velocity for this object is $v>200 \mathrm{~km} \mathrm{~s}^{-1}$. In this star, the photospheric nitrogen, oxygen and silicon are not underabundant, indicating a normal abundance pattern. Strangely enough, iron is clearly depleted, and this signature has not been wiped out. Altogether, fast rotation alone cannot account for the lack of $\lambda$ Boo stars in this sample; assuming an average inclination angle of $i=57^{\circ}$, only 2 sample sources (9\%) are expected to have rotational velocities above $125 \mathrm{~km} \mathrm{~s}^{-1}$.

HAEBE stars have ages of a few Myr and mass accretion is ongoing in a large fraction of these sources. When the dust particles in the circumstellar disk coagulate and grow, the coupling between gas and dust becomes smaller. Therefore, one intuitively expects that the $\lambda$ Bootis abundance pattern, which only occurs under stringent conditions, appears in the latest phase of the pre-main-sequence evolution of HAEBE stars (or later). During earlier stages, the mass infall might be too vicious, dragging along gas and dust. A necessary, but not satisfactory, 
condition for the depletion pattern to occur seems to be a quiet circumstellar environment, in which the dust has grown to appreciable size or disappeared, and a constant low-mass flow of depleted gas accretes onto the central star.

Vega-type stars are believed to be the successors of HAEBE stars. The circumstellar matter around these stars consists of the left-overs of the pre-main-sequence dust disk (cold particles at large distances to the central star and possibly a planetary system). None of our sample stars belonging to this group displays selective depletion of the metals. Gas accretion in most of these sources may have become too weak to produce a clear underabundance pattern.

HD 100546 is probably at the end of its pre-main-sequence lifetime. The observed $\lambda$ Bootis-like abundance pattern adds another pecularity to the list of curiosa (e.g., high degree of silicate crystallinity (Malfait et al. 1998b), spiral structure in the circumstellar disk (Grady et al. 2001)) known about this source.

\section{References}

Abt, H. A., \& Morrell, N. I. 1995, ApJS, 99, 135

Adelman, S. J. 1988, MNRAS, 230, 671

Adelman, S. J., Bolcal, C., Kocer, D., \& Hill, G. 1991, MNRAS, 252, 329

Anders, E., \& Grevesse, N. 1989, Geochim. Cosmochim. Acta, 53, 197

Augereau, J. C., Lagrange, A. M., Mouillet, D., \& Ménard, F. 2001, A\&A, 365, 78

Backman, D. E., \& Paresce, F. 1993, in Protostars and Planets III, 1253

Bell, K. R., \& Lin, D. N. C. 1994, ApJ, 427, 987

Beskrovnaya, N. G., Pogodin, M. A., Miroshnichenko, A. S., et al. 1999, A\&A, 343, 163

Bhatt, H. C., \& Manoj, P. 2000, A\&A, 362, 978

Blake, G. A., \& Boogert, A. C. A. 2004, ApJ, 606, L73

Chiang, E. I., \& Goldreich, P. 1997, ApJ, 490, 368

Cidale, L., Zorec, J., \& Tringaniello, L. 2001, A\&A, 368, 160

Coulson, I. M., Walther, D. M., \& Dent, W. R. F. 1998, MNRAS, 296, 934

Deliyannis, C. P., Boesgaard, A. M., Stephens, A., et al. 1998, ApJ, 498, L147

Dullemond, C. P. 2002, A\&A, 395, 853

Dullemond, C. P., \& Dominik, C. 2004, A\&A, 417, 159

Dullemond, C. P., Dominik, C., \& Natta, A. 2001, ApJ, 560, 957

Dunkin, S. K., Barlow, M. J., \& Ryan, S. G. 1997, MNRAS, 286, 604

Eggen, O. J. 1998, AJ, 115, 2397

Eisner, J. A., Lane, B. F., Akeson, R. L., Hillenbrand, L. A., \& Sargent, A. I. $2003, \mathrm{ApJ}, 588,360$

Erspamer, D., \& North, P. 2002, A\&A, 383, 227

Faraggiana, R., \& Bonifacio, P. 1999, A\&A, 349, 521

Faraggiana, R., Bonifacio, P., Caffau, E., Gerbaldi, M., \& Nonino, M. 2004, A\&A, 425, 615

Finkenzeller, U., \& Mundt, R. 1984, A\&AS, 55, 109

Flower, P. J. 1996, ApJ, 469, 355

Fuente, A., Rodríguez-Franco, A., Testi, L., et al. 2003, ApJ, 598, L39

Gerbaldi, M., Faraggiana, R., \& Lai, O. 2003, A\&A, 412, 447

Grady, C. A., Pérez, M. R., Thé, P. S., et al. 1995, A\&A, 302, 472

Grady, C. A., Polomski, E. F., Henning, T., et al. 2001, AJ, 122, 3396

Gray, R. O., \& Corbally, C. J. 1998, AJ, 116, 2530

Habing, H. J., Dominik, C., Jourdain de Muizon, M., et al. 2000, VizieR Online Data Catalog, 336, 50545
Heiter, U. 2002, A\&A, 381, 959

Herbig, G. H. 1960, ApJS, 4, 337

Hill, G. M. 1995, A\&A, 294, 536

Jenkins, E. 1989, in Interstellar Dust, IAU Symp., 135, 23

Kalas, P., Graham, J. R., Beckwith, S. V. W., Jewitt, D. C., \& Lloyd, J. P. 2002, ApJ, 567, 999

Kenyon, S. J., \& Hartmann, L. 1987, ApJ, 323, 714

Kessler, M. F., Steinz, J. A., Anderegg, M. E., et al. 1996, A\&A, 315, L27

Lagrange-Henri, A. M., Ferlet, R., Vidal-Madjar, A., et al. 1990, A\&AS, 85, 1089

Lambert, D. L., Hinkle, K. H., \& Luck, R. E. 1988, ApJ, 333, 917

Lemke, M. 1989, A\&A, 225, 125

Lemke, M. 1990, A\&A, 240, 331

Lin, D. N. C., \& Papaloizou, J. 1980, MNRAS, 191, 37

Malfait, K., Bogaert, E., \& Waelkens, C. 1998a, A\&A, 331, 211

Malfait, K., Waelkens, C., Waters, L. B. F. M., et al. 1998b, A\&A, 332, L25

Mannings, V., \& Sargent, A. I. 1997, ApJ, 490, 792

Marsakov, V. A., \& Shevelev, Y. G. 1995, Bulletin d'Information du Centre de Données Stellaires, 47, 13

Meeus, G., Waelkens, C., \& Malfait, K. 1998, A\&A, 329, 131

Meeus, G., Waters, L. B. F. M., Bouwman, J., et al. 2001, A\&A, 365, 476

Miroshnichenko, A. S., Mulliss, C. L., Bjorkman, K. S., et al. 1999, MNRAS, 302, 612

Molster, F. J., Waters, L. B. F. M., Trams, N. R., et al. 1999, A\&A, 350,163

Mora, A., Merín, B., Solano, E., et al. 2001, A\&A, 378, 116

Natta, A., Testi, L., Neri, R., Shepherd, D. S., \& Wilner, D. J. 2004, A\&A, 416, 179

Pantin, E., Waelkens, C., \& Lagage, P. O. 2000, A\&A, 361, L9

Patten, B. M., \& Willson, L. A. 1991, AJ, 102, 323

Paunzen, E., Andrievsky, S. M., Chernyshova, I. V., et al. 1999, A\&A, 351,981

Pérez, M. R., \& Grady, C. A. 1997, Space Sci. Rev., 82, 407

Piétu, V., Dutrey, A., \& Kahane, C. 2003, A\&A, 398, 565

Qiu, H. M., Zhao, G., Chen, Y. Q., \& Li, Z. W. 2001, ApJ, 548, 953

Renson, P., Faraggiana, R., \& Boehm, C. 1990, Bulletin d'Information du Centre de Données Stellaires, 38, 137

Santos, N. C., Israelian, G., \& Mayor, M. 2001, A\&A, 373, 1019

Smith, K. C., \& Dworetsky, M. M. 1993, A\&A, 274, 335

Sneden, C. A. 1973, Ph.D. Thesis

Song, I., Caillault, J.-P., Barrado y Navascués, D., Stauffer, J. R., \& Randich, S. 2000, ApJ, 533, L41

Takeda, Y., Takada-Hidai, M., Jugaku, J., Sakaue, A., \& Sadakane, K. 1999, PASJ, 51, 961

Testi, L., Palla, F., \& Natta, A. 1998, A\&AS, 133, 81

Testi, L., Natta, A., Shepherd, D. S., \& Wilner, D. J. 2003, A\&A, 403, 323

Thé, P. S., de Winter, D., \& Pérez, M. R. 1994, A\&AS, 104, 315

Turcotte, S., \& Charbonneau, P. 1993, ApJ, 413, 376

van den Ancker, M. E., de Winter, D., \& Tjin A Djie, H. R. E. 1998, A\&A, 330, 145

van Winckel, H. 2003, ARA\&A, 41, 391

van Winckel, H., Waelkens, C., \& Waters, L. B. F. M. 1995, A\&A, 293, L25

Venn, K. A., \& Lambert, D. L. 1990, ApJ, 363, 234

Waters, L. B. F. M., Beintema, D. A., Zijlstra, A. A., et al. 1998, A\&A, 331, L61

Waters, L. B. F. M., Trams, N. R., \& Waelkens, C. 1992, A\&A, 262, L37

Waters, L. B. F. M., \& Waelkens, C. 1998, ARA\&A, 36, 233 
B. Acke and C. Waelkens: Elemental abundances of young stars, Online Material $p 1$

\section{Online Material}




\section{Appendix: Notes on individual objects}

HD 6028. Stellar parameters come from Coulson et al. (1998). HD 17081. Adelman et al. (1991) determined the iron abundance $[\mathrm{Fe}]=-0.07$ dex. Takeda et al. (1999) determined $[\mathrm{O}]=-0.14 \mathrm{dex},[\mathrm{Fe}]=-0.02 \mathrm{dex}$ and $v \sin i=19.2 \mathrm{~km} \mathrm{~s}^{-1}$. Our iron abundance is $\sim 0.20$ dex lower. The oxygen abundance and the projected rotational velocity are in good agreement with our values. Smith \& Dworetsky (1993) suggested $[\mathrm{Cr}]=+0.33 \mathrm{dex},[\mathrm{Fe}]=-0.02 \mathrm{dex}$ and $[\mathrm{Ni}]=-0.05$ dex. Only the nickel abundance is in agreement with our determinations. HD 17206. Marsakov \& Shevelev (1995) derived an iron abundance of -0.07 dex. This is in excellent agreement with our results.

HD 18256. Eggen (1998) computed $[\mathrm{Fe}]=+0.13$ dex which is fairly close to our determination.

HD 20010. Santos et al. (2001) and Marsakov \& Shevelev (1995) derived respectively $[\mathrm{Fe}]=-0.20$ dex and $[\mathrm{Fe}]=-0.23 \mathrm{dex}$. This strokes with our results.

HD 28978. The computed abundances of Lemke $(1989,1990)$ $[\mathrm{CI}]=-0.05 \mathrm{dex},[\mathrm{Si} \mathrm{II}]=-0.06 \mathrm{dex},[\mathrm{CaI}]=-0.07 \mathrm{dex}$, $[\mathrm{Ti}$ II $]=+0.15 \operatorname{dex},[\mathrm{Fe} \mathrm{I}]=+0.07 \mathrm{dex},[\mathrm{Fe} \mathrm{II}]=+0.19 \mathrm{dex}$ and $[\mathrm{Ba} I \mathrm{II}]=+0.61 \mathrm{dex}$ are in fairly good agreement with our results. The result for the heavy element strontium differs from our determinations: $[\mathrm{Sr} \mathrm{II}]_{\text {Lemke }}=+0.55 \mathrm{dex}$ versus our $[\mathrm{Sr}$ II $]=+0.31 \mathrm{dex}$.

HD 31648. Stellar parameters are taken from van den Ancker et al. (1998). Our measured projected velocity agrees reasonably with the value $v \sin i=102 \mathrm{~km} \mathrm{~s}^{-1}$ of Mora et al. (2001).

HD 33564. Deliyannis et al. (1998) suggested $[\mathrm{Fe}]=-0.05$ dex. Marsakov \& Shevelev (1995) derived $[\mathrm{Fe}]=-0.11$ dex. We find a stronger underabundance of iron, $[\mathrm{Fe} \mathrm{I}]=-0.35 \mathrm{dex}$ and $[\mathrm{Fe} \mathrm{II}]=-0.41 \mathrm{dex}$.

HD 36112. Beskrovnaya et al. (1999) determined $[\mathrm{Si}] \approx+0.40 \mathrm{dex},[\mathrm{Ca}]=+0.06 \mathrm{dex},[\mathrm{Fe}]=-0.02 \mathrm{dex}$ and $v \sin i=60 \mathrm{~km} \mathrm{~s}^{-1}$. The calcium and iron abundances and the projected rotational velocity are consistent with our findings. The silicon abundance of Beskrovnaya et al. is $\sim 0.40 \mathrm{dex}$ higher than our value.

HD 95418. Hill (1995) derived $[\mathrm{Fe}]=+0.14$ dex which is comparable to our results.

HD 97048. Stellar parameters come from van den Ancker et al. (1998). Because of the large projected rotational velocity ( $v \sin i=140 \mathrm{~km} \mathrm{~s}^{-1}$ ) the equivalent widths of the spectral lines are difficult to determine. We based our oxygen abundance computation on two O I multiplets at $6156 \AA$ and $6454 \AA$.

HD 97633. Lemke $(1989,1990)$ determined $[\mathrm{Si} I \mathrm{II}]=+0.15$ $\operatorname{dex},[\mathrm{Ca} I]=+0.17 \mathrm{dex},[\mathrm{Ti} \mathrm{II}]=+0.20 \mathrm{dex},[\mathrm{Fe} \mathrm{I}]=+0.24 \mathrm{dex}$ and $[\mathrm{Fe} \mathrm{II}]=+0.30 \mathrm{dex}$. The silicon abundance is in good agreement with our value. The other elemental abundances are systematically $\sim 0.20$ dex higher than our results. Our iron abundance $([\mathrm{Fe}]=+0.02 \mathrm{dex})$ confirms the result of Adelman (1988). Takeda et al. (1999) computed $[\mathrm{O}]=-0.27 \mathrm{dex}$, $[\mathrm{Fe}]=+0.19 \mathrm{dex},[\mathrm{Si}]=-0.43 \mathrm{dex}$ and $v \sin i=21.6 \mathrm{~km} \mathrm{~s}^{-1}$. There is a discrepancy with our silicon and iron abundances. Smith \& Dworetsky (1993) determined $[\mathrm{Cr}]=+0.43$ dex and $[\mathrm{Fe}]=+0.03 \mathrm{dex}$. We find a chromium abundance of $+0.17 \mathrm{dex}$. HD 100453. Stellar parameters were taken from Meeus et al. (2001). We altered the $\log g$ value from 4.5 to 4.0. About 180 spectral lines were used in the abundance determinations.

HD 102647. Stellar parameters were extracted from Habing et al. (2000).

HD 104237. We took the stellar parameters of Meeus et al. (2001). Close to 440 lines were included in the abundance computations.

HD 135344. We adopted the gravity parameter of Meeus et al. (2001) from $\log g=4.5$ down to 4.0. We kept $T_{\mathrm{e}}$ from that article. The projected rotational velocity is $80 \mathrm{~km} \mathrm{~s}^{-1}$. We specifically looked for unblended spectral lines by comparing the spectrum of HD 135344 with the spectra of HD 18256 and HD 20010 , two stars with a similar effective temperature, but much lower $v \sin i$. By doing this we diminish the risk of misidentifying lines and measuring wrong equivalent widths.

HD 190073. Based on the A3IIIe spectral classification by Gray \& Corbally (1998) and the A2IVe classification by Mora et al. (2001) we chose an effective temperature $T_{\mathrm{e}}=9250 \mathrm{~K}$ and a gravity $\log g=3.5$ for the Kurucz model. Other articles suggested a much higher effective temperature (e.g. $11120 \mathrm{~K}$ and a B9IV classification, Cidale et al. 2001; or A0IV by Thé et al. 1994). Nevertheless, the lower temperature was consistent with the spectrum of HD 190073 (see Fig. 4). Some of the spectral lines of Ca I, Ca II, Sc II, Ti II, Cr II, Fe I and Fe II depict emission features. The emission fills the core of the photospheric absorption line. Hence, the equivalent width of the line cannot be measured accurately. The spectral lines that display emission are therefore not included in the abundance analysis of HD 190073.

HD 244604. The stellar parameters of HD 244604 are extracted from Miroshnichenko et al. (1999).

HD 245185. Not one single absorption line was detected. Our signal-to-noise level in this spectrum was about $S / N \sim 30$, which makes it by far the worst spectrum in our sample. The projected rotational velocity could not be derived. Stellar parameters for HD 245185 come from Testi et al. (1998).

HD 250550. Stellar parameters were taken from Testi et al. (1998). 